\title{
The Significance of Plasma Lysophospholipids in Patients with Renal Failure on Hemodialysis
}

\author{
Takayo Sasagawa, ${ }^{1}$ Kazuhiko Suzuki, ${ }^{1}$ Tetsuya Shiota, ${ }^{2}$ \\ Tadaaki KonDO $^{3}$ and Misako OKITA ${ }^{1, *}$ \\ ${ }^{1}$ Department of Nutritional Science, Faculty of Health and Welfare Science, \\ Okayama Prefectural University, Soja 719-1197, Japan \\ ${ }^{2}$ Division of Internal Medicine, Hukuwatari Hospital, \\ Takebe 709-3111, Japan \\ ${ }^{3}$ Division of Internal Medicine, Shigei Hospital, \\ Kurashiki 710-0051, Japan
}

(Received April 28, 1998)

Summary Abnormal phospholipid metabolism may play an important role in the progression of atherosclerosis in renal failure. We analyzed plasma phospholipid and lysophospholipid contents and fatty acid composition in the phospholipids of 18 patients with renal failure on hemodialysis (HD) and compared the levels with those of healthy controls. HD patients had a notably higher molar ratio of the plasma lysophosphatidylcholine (lysoPC)/phosphatidylcholine (PC) distributed from 0.072 to 0.207 , and the control group showed a ratio lower than 0.150 . Plasma phosphatidylethanolamine (PE) concentration significantly increased in HD patients compared with control subjects. A much higher level of plasma lysophosphatidic acid (lysoPA) $(1.41 \pm 0.16 \mathrm{nmol} / \mathrm{mL})$ was observed in HD patients compared with controls $(0.54 \pm 0.08 \mathrm{nmol} / \mathrm{mL})$. A strong correlation was found between plasma lysoPA and lysoPC concentrations $(r=0.609, p<0.01)$ in HD patients. Serum inorganic phosphate $(\mathrm{P})$ concentration was associated with the abnormal plasma lysoPC/PC, PE, and lysoPA levels in HD patients. Important decreases in eicosapentaenoic acid (EPA) of plasma PC and of dietary intake were observed in HD patients. Plasma thiobarbituric acid reactive substance (TBARS) concentration was negatively correlated with the amount of vitamin $E$ intake in both subjects. These findings demonstrated the specific characteristics of the abnormal phospholipid metabolism in HD patients. The cause and consequences of elevated lysoPC/PC molar ratio and lysoPA in the plasma of HD patients remain to be established.

Key Words lysophospholipid, plasma lipid, dietary fat intake, renal failure, hemodialysis

\footnotetext{
*To whom correspondence should be addressed. E-mail: okita@fhw.oka-pu.ac.jp
} 
Changes in lipid metabolism during chronic renal failure have been associated with a high mortality rate from atherosclerosis (1). Hypertriglyceridemia and low plasma high-density lipoprotein (HDL)-cholesterol $(2,3)$ have been commonly observed in patients with chronic renal failure. It was proposed that abnormal lipid metabolism, increased intermediate density lipoprotein (IDL), and decreased HDL levels may promote atherosclerosis in uremia (4). On the other hand, oxidized lowdensity lipoprotein (LDL) has been emphasized as a likely atherogenic lipoprotein (5). Oxidized LDL-induced growth of macrophage required lysophosphatidylcholine (lysoPC) (6). Indeed, enhanced LDL oxidation was shown in uremic patients (7). LysoPC can be generated from phosphatidylcholine (PC) through the action of plasma lecithin cholesterol acyltransferase (LCAT) or phospholipases. Therefore abnormal metabolism in phospholipids is expected to play an important role in the progression of atherogenic lesion in renal failure.

Fatty acid compositions of phospholipids in the cell membrane are very important, since polyunsaturated fatty acids are converted to various eicosanoids. Because polyunsaturated fatty acids are highly sensitive to oxidative stress, the membrane phospholipids rich in polyunsaturated fatty acids are more oxydizable than those low in these fatty acids. The polyunsaturated fatty acid composition is altered mainly with a dietary fatty acid.

Taking these points into consideration, we analyzed plasma phospholipid and lysophospholipid contents and fatty acid compositions in these lipids in patients with renal failure on hemodialysis (HD).

\section{METHODS}

Subjects. Eighteen patients with chronic renal failure, aged 26 to 79 (mean \pm SE 56 $\pm 3,7$ males and 11 females), who were undergoing stable maintenance HD participated in this study. The duration of maintenance hemodialysis treatment was 6 to 267 months (mean \pm SE $123 \pm 20$ ). The etiology of chronic renal failure consisted of chronic glomerulonephritis (14 patients), hypertensive nephrosclerosis (1), renal tuberculosis (1), or unknown (2). One patient had retinal angiosclerosis, and another had retinal hemorrhage. None of the patients had the events of coronary ischemic diseases, cerebral vascular diseases, or arteriosclerosis obliterans. Diabetic patients were excluded from the subjects. Fifteen healthy volunteers aged 30 to 60 (46 $\pm 2,5$ males and 10 females) were recruited as controls. The mean body mass indexes (BMI) in HD patients and control subjects were $19.5 \pm 0.5$ and $22.4 \pm 0.4$, respectively. This study complied with the code of ethics of the World Medical Association (Declaration of Helsinki), and informed consent was obtained from all subjects.

Analytical methods. Blood samples were taken from the efferent line before hemodialysis and were immediately mixed with ethylenediaminetetraacetic acid (EDTA) preventing coagulation and phospholipase activity. Plasma was separated after the centrifugation of blood samples at $1,000 \times g$ for $15 \mathrm{~min}$ at $4^{\circ} \mathrm{C}$ and stored 
Table 1. Plasma lipids in HD patients and control subjects.

\begin{tabular}{lcc}
\hline & $\begin{array}{c}\text { Controls } \\
(n=15)\end{array}$ & $\begin{array}{c}\text { HD patients } \\
(n=18)\end{array}$ \\
\hline Triglyceride $(\mathrm{mg} / \mathrm{dL})$ & $81 \pm 9$ & $105 \pm 12$ \\
Total cholesterol $(\mathrm{mg} / \mathrm{dL})$ & $191 \pm 11$ & $162 \pm 9^{*}$ \\
HDL cholesterol $(\mathrm{mg} / \mathrm{dL})$ & $74 \pm 6$ & $44 \pm 2^{* *}$ \\
Total cholesterol/HDL cholesterol & $2.88 \pm 0.21$ & $3.70 \pm 0.14^{* *}$ \\
\hline
\end{tabular}

Mean \pm SE, ${ }^{* *} p<0.01, * p<0.05$

at $-80^{\circ} \mathrm{C}$ until assayed.

Plasma lipids were extracted by the method of Bligh and Dyer (8) and phospholipids were separated by 2-dimensional thin-layer chromatography (TLC) as described in our previous reports (9). The spots corresponding to PC, phosphatidylethanolamine (PE), phosphatidylinositol (PI), lysoPC, and lysophosphatidic acid (lysoPA) were scraped from the TLC plates and transmethylated by using acetyl chloride/methanol 5:50 (v/v). A known amount of heptadecanoic acid was used as an internal standard. Following transmethylation, fatty acid methyl esters were extracted with petroleum ether and quantified by using a model GC14A gas chromatograph (Shimadzu, Kyoto, Japan). Concentrations of each phospholipid presented herein were derived from the fatty acid content assessed by gas chromatography and calibrated with heptadecanoic acid. Plasma thiobarbituric acid reactive substances (TBARS) were measured as an indicator of lipid peroxidation with a commercial kit (Wako Pure Chemical Industries, Osaka, Japan) based on the method of Yagi (10).

The dietary intake of energy, protein, fat, vitamin $E$, and fatty acids was calculated by means of the dietary records for $3 \mathrm{~d}$ before blood sampling.

Statistical analysis. Data were presented as means \pm SE. For comparison between the 2 groups, patients and controls, the Mann-Whitney $U$-test was used. The Spearman $R$-test was used to calculate correlation coefficients.

\section{RESULTS}

\section{Plasma lipid concentration}

Total cholesterol and HDL cholesterol were significantly decreased in HD patients (Table 1). Hypertriglyceridemia was observed in only 4 patients, and the mean triglyceride (TG) level in HD patients was not greatly higher than in controls. The atherogenic index (total cholesterol/HDL cholesterol) was much higher in HD patients than in controls.

A notable decrease in plasma PC and lysoPC and a notable increase in PE were observed in HD patients compared with controls (Table 2). Plasma PI concentration showed no significant change. Although the lysoPC concentration 
Table 2. Plasma phospholipids in HD patients and control subjects.

\begin{tabular}{|c|c|c|}
\hline & $\begin{array}{l}\text { Controls } \\
(n=15)\end{array}$ & $\begin{array}{l}\text { HD patients } \\
\quad(n=18)\end{array}$ \\
\hline Phosphatidylcholine (nmol/mL) & $1,214 \pm 59$ & $887 \pm 49^{* * *}$ \\
\hline Phosphatidylethanolamine $(\mathrm{nmol} / \mathrm{mL})$ & $48.6 \pm 3.0$ & $60.5 \pm 4.2^{*}$ \\
\hline Phosphatidylinositol (nmol/mL) & $55.1 \pm 5.1$ & $47.1 \pm 2.9$ \\
\hline Lysophosphatidylcholine (nmol/mL) & $133 \pm 5$ & $115 \pm 7^{*}$ \\
\hline Lysophosphatidic acid (nmol/mL) & $0.54 \pm 0.08$ & $1.41 \pm 0.16^{* * *}$ \\
\hline LysoPC/PC & $0.11 \pm 0.01$ & $0.13 \pm 0.01^{*}$ \\
\hline
\end{tabular}

Mean $\pm \mathrm{SE},{ }^{* * *} p<0.001,{ }^{*} p<0.05$.

PC: phosphatidylcholine.

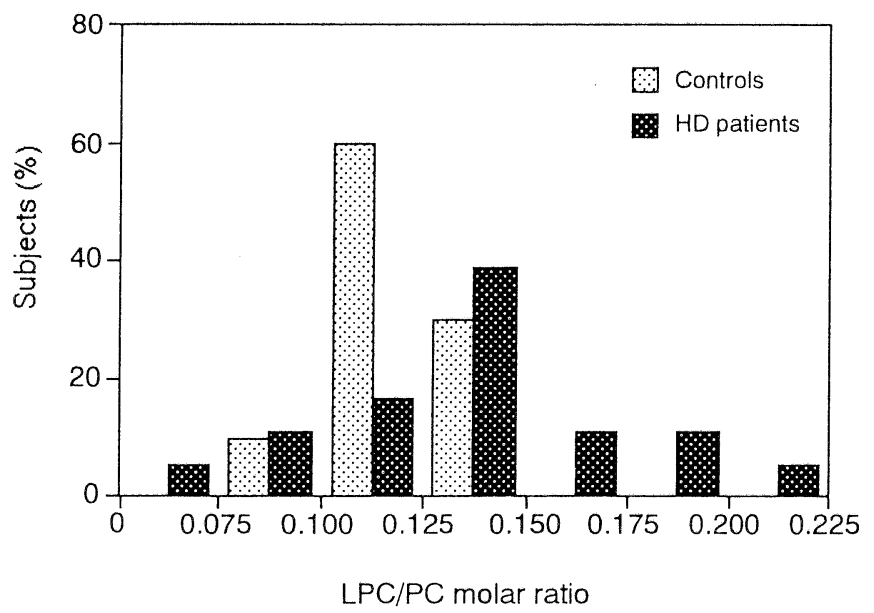

Fig. 1. Distribution of plasma lysoPC/PC molar ratio in HD patients and control subjects.

was decreased, the lysoPC/PC molar ratio in $\mathrm{HD}$ patients was much higher than in controls. The lysoPC/PC ratio in the control subjects showed a level lower than 0.150 ; it also showed the level in $60 \%$ of the subjects to be distributed from 0.101 to 0.125 (Fig. 1). In contrast to controls, the ratio in HD patients was distributed from 0.072 to 0.207 , and 5 of 18 patients showed a level higher than 0.150 , which was the highest level in controls. Two patients having atherosclerosis lesions of retinal blood vessels showed ratios of 0.180 and 0.151 . The lysoPC/PC molar ratio of HD patients correlated positively with serum inorganic phosphate (P) concentration $(r=0.520, p<0.05)$. Furthermore, the $\mathrm{PC} / \mathrm{PE}$ molar ratio in HD patients correlated negatively with serum $\mathrm{P}$ concentration $(r=-0.725, p<0.002)$ and positively with serum calcium $(\mathrm{Ca}) / \mathrm{P}$ ratio $(r=0.853, p<0.0001)$. A negative and weighty correlation $(r=-0.579, p<0.02)$ between plasma PC and creatinine 
concentrations was recognized in HD patients.

A notably higher level of plasma lysoPA was observed in HD patients compared with control subjects. A significant correlation $(r=0.609, p<0.01)$ was recognized between the plasma lysoPA and lysoPC concentrations only in HD patients (Fig. 2). Serum $\mathrm{Ca} / \mathrm{P}$ ratio tended to correlate negatively with plasma lysoPA concentration $(r=-0.452, p<0.10)$.

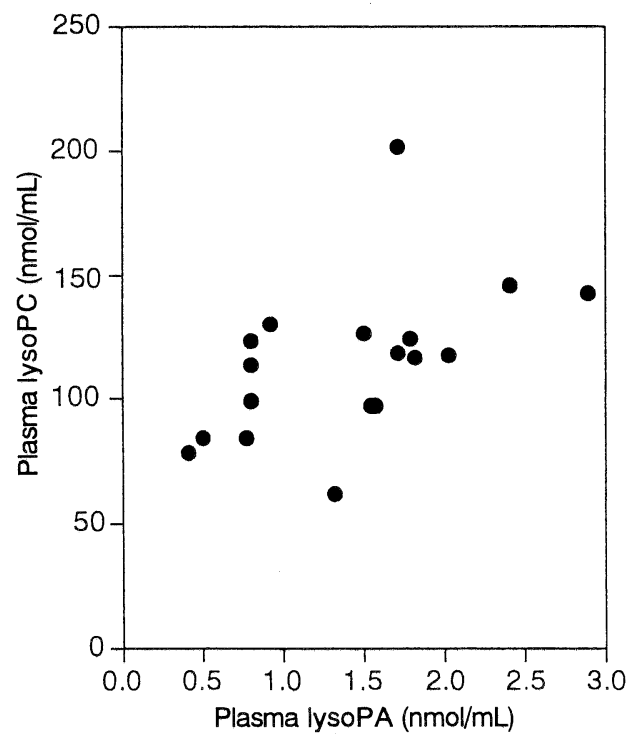

Fig. 2. Correlation between plasma lysoPC and lysoPA concentrations in HD patients $(r=0.609, p<0.01)$.

Table 3. Fatty acid composition of plasma phosphatidylcholine and lysophosphatidylcholine in HD patients and control subjects.

\begin{tabular}{lccccc}
\hline & \multicolumn{2}{c}{ Phosphatidylcholine } & & \multicolumn{2}{c}{ Lysophosphatidylcholine } \\
\cline { 2 - 3 } & $\begin{array}{c}\text { Controls } \\
(n=15)\end{array}$ & $\begin{array}{c}\text { HD patients } \\
(n=18)\end{array}$ & & $\begin{array}{c}\text { Controls } \\
(n=15)\end{array}$ & $\begin{array}{c}\text { HD patients } \\
(n=18)\end{array}$ \\
& $31.9 \pm 0.3$ & $33.3 \pm 0.5^{*}$ & & $47.8 \pm 1.1$ & $53.9 \pm 1.0^{*}$ \\
& $14.9 \pm 0.4$ & $13.9 \pm 0.5$ & & $18.1 \pm 0.4$ & $18.1 \pm 0.8$ \\
Palmitic acid & $11.1 \pm 0.5$ & $12.4 \pm 0.3^{*}$ & & $9.7 \pm 0.4$ & $10.8 \pm 0.5$ \\
Stearic acid & $21.7 \pm 1.2$ & $23.0 \pm 0.7$ & & $15.4 \pm 0.8$ & $12.8 \pm 0.8$ \\
Oleic acid & $6.4 \pm 0.3$ & $6.6 \pm 0.3$ & & $1.9 \pm 0.1$ & $1.7 \pm 0.1$ \\
Linoleic acid & $3.6 \pm 0.6$ & $2.1 \pm 0.2^{*}$ & & $0.9 \pm 0.2$ & $0.8 \pm 0.1$ \\
Arachidonic acid & $5.6 \pm 0.6$ & $4.7 \pm 0.3$ & & $1.0 \pm 0.2$ & $1.1 \pm 0.1$ \\
Eicosapentaenoic acid & (mol \%) &
\end{tabular}

Mean \pm SE, $* p<0.05$

Vol 44, No 6, 1998 
Fatty acid compositions in PC and lysoPC

A sizable increase of palmitic and oleic acids and a decrease of eicosapentaenoic acid (EPA) were recognized in the plasma PC of HD patients compared with those of controls (Table 3). In the lysoPC species, palmitoyl-lysoPC is the primary one, and the percentage of palmitoyl-lysoPC (in terms of mol \% of total fatty acids) in HD patients was much higher than that of controls.

Table 4. Dietary lipids ingested in HD patients and control subjects.

\begin{tabular}{ccc}
\hline & $\begin{array}{c}\text { Controls } \\
(n=15)\end{array}$ & $\begin{array}{c}\text { HD patients } \\
(n=18)\end{array}$ \\
\hline Total fatty acids $(\mathrm{g} / \mathrm{d})$ & $39.1 \pm 2.1$ & $33.6 \pm 2.3$ \\
Saturated fatty acid (\%) & $31.8 \pm 1.2$ & $25.4 \pm 0.8^{* *}$ \\
Monounsaturated fatty acid (\%) & $39.7 \pm 0.7$ & $42.8 \pm 0.7^{*}$ \\
Polyunsaturated fatty acid (\%) & $28.5 \pm 0.9$ & $31.9 \pm 0.9^{*}$ \\
Linoleic acid (g/d) & $8.7 \pm 0.5$ & $8.4 \pm 0.6$ \\
$\alpha$-Linolenic acid (g/d) & $1.3 \pm 0.1$ & $1.5 \pm 0.1$ \\
Eicosapentaenoic acid (g/d) & $0.28 \pm 0.05$ & $0.17 \pm 0.04^{*}$ \\
Docosahexaenoic acid (g/d) & $0.52 \pm 0.09$ & $0.34 \pm 0.07^{*}$ \\
$n$-6/n-3 fatty acid ratio & $4.2 \pm 0.4$ & $4.3 \pm 0.2$ \\
Vitamin E (mg/d) & $6.7 \pm 0.4$ & $5.9 \pm 0.4$ \\
Vitamin E/PUFA (mg/g) & $0.61 \pm 0.03$ & $0.56 \pm 0.02$ \\
Cholesterol (mg/d) & $224 \pm 27$ & $236 \pm 32$ \\
\hline
\end{tabular}

Mean $\pm \mathrm{SE},{ }^{* *} p<0.01, * p<0.05$

PUFA: polyunsaturated fatty acid.

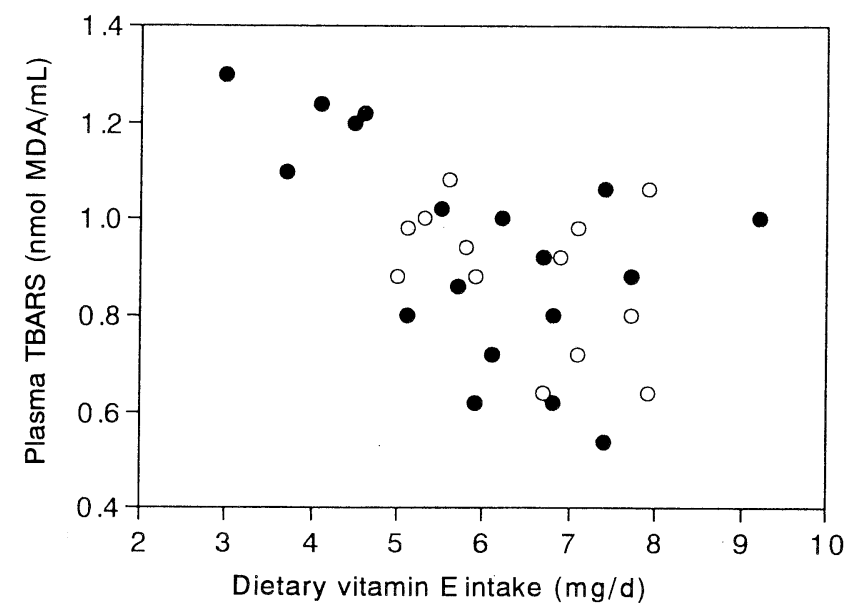

Fig. 3. Correlation between dietary vitamin $E$ intake and plasma TBARS in HD patients $(\bigcirc)$ and control subjects $(\bigcirc)(r=-0.383, p<0.05)$. 


\section{Dietary intake of fatty acids and vitamin $E$}

HD patients and controls showed no important differences in energy, protein, and fat intakes. EPA and docosahexaenoic acid (DHA) intakes were notably lower in HD patients than in controls (Table 4). The intake of vitamin E and of cholesterol in HD patients was similar to those in controls. Dietary linoleic acid intake markedly correlated with the linoleic acid content in plasma PC in HD patients $(r=0.767$, $p<0.001$ ).

Plasma TBARS concentration was negatively correlated with the amount of vitamin E intake (Fig. 3).

\section{DISCUSSION}

In the present study, we recognized a higher lysoPC/PC molar ratio in the plasma of renal failure patients on HD in spite of low plasma PC and lysoPC concentrations. The low plasma PC and lysoPC concentrations may reflect in part a low serum lipoprotein concentration, because the plasma lysoPC and PC concentrations correlated significantly with the serum total cholesterol concentration in the present study ( $r=0.552, p<0.02$, and $r=0.467, p<0.05$, respectively). The elevated lysoPC/PC molar ratio may suggest an enhanced formation of lysoPC or a limited tissue uptake of the lipid. The most likely source of plasma lysoPC is fatty acid release, mainly polyunsaturated fatty acid (PUFA) such as arachidonic acid, from the sn-2 position of PC. Increased plasma lecithin: cholesterol acyltransferase (LCAT) or phospholipase (PL) $\mathrm{A}_{2}$ activity would be compatible with the elevated plasma level of lysoPC rich in palmitic acid. However, decreased plasma LCAT activity was recognized in renal failure (11). On the other hand, Costello et al (12) demonstrated that plasma PLA 2 activity not only was increased eightfold in uremic patients, but also was less sensitive to inhibition by heparin compared with that of normal subjects. Therefore a role for $\mathrm{PLA}_{2}$ in the elevation of plasma lysoPC/PC molar ratio is implied in HD patients; moreover, the low plasma PC level in HD patients may be a result of an increased PLA 2 hydrolysis of PC.

PC is formed from PE by transmethylation. An increase in plasma homocystein and an erythrocyte accumulation of S-adenosylhomocysteine, a potent inhibitor of most methyltransferases, were reported in HD patients (13). Thus the increased PE concentration and low $\mathrm{PC} / \mathrm{PE}$ ratio in the present $\mathrm{HD}$ patients may be explained by an inhibition of transmethylation. An inhibition of transmethylation may be another explanation for the low plasma PC concentration in HD patients. Creatinine, a uremic toxin, is also supposed to have some deleterious effects on phosphatidylcholine metabolism, though the mechanism is unknown.

LysoPC and free fatty acids produced from PC hydrolysis are both implicated in signal transduction. LysoPC is known to exert a variety of biological activities, such as T-lymphocyte activation (14) and enhancement of adhesion molecule expression in endothelial cells (15), and it was reported to play an important role 
in mitogenic activity of oxidized LDL for human monocyte-derived macrophages (16). The uptake of oxidized LDL by macrophages may stimulate it to produce growth factors, cytokines, and other mediators. In the several lysoPC species tested, palmitoyl-lysoPC most potentiated the diacylglycerol-induced activation of protein kinase $\mathrm{C}$ in vitro (17). Although the plasma lysoPC concentration in HD patients was lower, the percentage of palmitoyl-lysoPC was higher than in controls, and no significant difference was noted in plasma palmitoyl-lysoPC concentration between HD patients and controls. In HD patients having a higher serum lysoPC concentration, therefore, the biological significance of lysoPC may be higher than in control subjects.

LysoPA is known as a biological modulator of cellular function, and has reportedly been produced from PI via diacylglycerol and phosphatidic acid (PA). LysoPA is a product of PLA 2 hydrolysis of PA. It was also suggested, however, that lysoPA may be provided from lysoPC through hydrolysis by lysophospholipase D (lysoPLD) (18). Both PLA 2 and lysoPLD are possible causes for the elevated level of plasma lysoPA in the present HD patients because increased plasma $\mathrm{PLA}_{2}$ activity was suggested in uremic patients (12), and lysoPA concentration correlated significantly with serum lysoPC concentration (Fig. 2). LysoPA is rapidly produced in thrombin-activated platelets (19) and increases during blood clotting. The lysoPA concentrations in the plasma obtained before and after HD in 4 patients were then compared, and no important differences were recognized $(1.47 \pm 0.41$ and $1.91 \pm 0.06$, respectively). However, Cases et al (20) reported that cellulosic membranes induced platelet activation during HD. Therefore it is undeniable that activated platelets produced lysoPA during HD in the present study.

Serum $\mathrm{P}$ concentration was associated with increased PI, PE, lysoPC, and lysoPA concentrations and with elevated lysoPC/PC ratio. Thus our present results may be supported by the finding of Nishizawa et al (21), which suggests that hyperphosphatemia and hyperparathyroidism affect the lipoprotein metabolism in HD patients. Hyperphosphatemia may also affect the phospholipid metabolism in HD patients.

Dietary fatty acid is an important factor affecting the composition of fatty acids in plasma and tissues. The linoleic acid molar ratio in plasma PC correlated significantly with linoleic acid intake in the present HD patients and in controls. The substitution of EPA for arachidonic acid reportedly reduced the production of eicosanoids, especially thromboxanes (TXs) (22). The lower intake of EPA in HD patients might have some promoting effects on TX production, but it was not examined in the present study.

Elevated levels of lipid peroxidation products have been found in red cell membranes from uremic patients (23). Some evidence exists that hemodialysis per se may be an oxidant stress resulting from neutrophile activation, degranulation, and uncontrolled release of reactive oxygen species into the circulation (24). It was shown that mononuclear leukocytes from chronic renal failure patients and from hemodialysis patients had increased susceptibility to membrane lipid peroxidation 
(25). The treatment of HD patients with vitamin $E(300 \mathrm{mg} / \mathrm{d})$ for 1 month resulted in a significant decrease of lipid peroxidation (23). Our present data also indicate a beneficial effect of vitamin $E$ intake on the prevention of lipid peroxidation in HD patients.

We conclude that the lysoPC/PC molar ratio and lysoPA, which were elevated in the plasma of HD patients, should have some detrimental effects on the progression of atherogenic complications during chronic hemodialysis and that EPA and vitamin E supplements may have some beneficial effects on suppressing the progression.

\section{REFERENCES}

1) Lindner A, Charra B, Sherrard D, Scribner B. 1974. Accelerated atherosclerosis in prolonged maintenance hemodialysis. New Engl J Med 290: 697-701.

2) McCosh EJ, Solangi K, Rivers JM, Goodman A. 1975. Hypertriglyceridemia in patients with chronic renal failure. Am J Clin Nutr 28: 1036-1043.

3) Avram MM, Goldwasser P, Burrell DE, Antignani A, Fein PA, Mittman N. 1992. The uremic dyslipidemia: A cross-sectional and longitudinal study. Am J Kidney Dis 20: 324-335.

4) Shoji $T$, Nishizawa $Y$, Kawagishi $T$, Tanaka M, Kawasaki $K$, Tabata $T$, Inoue $T$, Morii H. 1997. Atherogenic lipoprotein changes in the absence of hyperlipidemia in patients with chronic renal failure treated by hemodialysis. Atherosclerosis 131: 229-236.

5) Ylä-Herttuala S, Palinski W, Rosenfeld ME, Parthaserthy S, Carew TE, Butler S, Witztum JL, Steinberg D. 1989. Evidence for the presence of oxidatively modified low density lipoprotein in atherosclerotic lesions of rabbit and man. $J$ Clin Invest 84 : 1086-1095.

6) Sakai M, Miyazaki A, Hakamata H, Sasaki T, Yui S, Yamazaki M, Shichiri H, Horiuchi S. 1994. Lysophosphatidylcholine plays an essential role in the mitogenic effect of oxidized low density lipoprotein on murine macrophages. J Biol Chem 269: 31430-31435.

7) Maggi E, Bellazzi R, Falaschi F, Frattoni A, Perani G, Finardi G, Gazo A, Nai M, Romanini D, Bellomo G. 1994. Enhanced LDL oxidation in uremic patients: An additional mechanism for accelerated atherosclerosis? Kidney Int 45: 876-883.

8) Bligh EG, Dyer WJ. 1959. A rapid method of total lipid extraction and purification. Can J Biochem Physiol 37: 911-917.

9) Okita M, Gaudette DC, Mills GB, Holub BJ. 1997. Elevated levels and altered fatty acid composition of plasma lysophosphatidylcholine (lysoPC) in ovarian cancer patients. Int J Cancer 71: 31-34.

10) Yagi K. 1982. Assay for serum lipid peroxide and its clinical significance. In: Lipid Peroxides in Biology and Medicine (Yagi K, ed), p 223-242. Academic Press, New York.

11) McLeod R, Reeve CE, Frohlich J. 1984. Plasma lipoproteins and lecithin:cholesterol acyltransferase distribution in patients receiving maintenance hemodialysis. Kidney Int 25: 683-688.

12) Costello J, Franson RC, Landwehr K, Landwhr DM. 1990. Activity of phospholipase $\mathrm{A}_{2}$ in plasma increases in uremia. Clin Chem 36: 198-200.

13) Perna AF, Ingrosso D, De Santo NG, Galletti P, Zappia V. 1995. Mechanism of erythrocyte accumulation of methylation inhibitor S-adenosylhomocysteine in uremia. Kidney Int 47: 247-253. 
14) Asaoka Y, Oka M, Yoshida K, Sasaki Y, Nishizuka Y. 1992. Role of lysophosphatidylcholine in T-lymphocyte activation: involvement of phospholipase $\mathrm{A}_{2}$ in signal transduction through protein kinase C. Proc Natl Acad Sci USA 89: 6447-6451.

15) Yokote K, Morisaki N, Zenibayashi M, Ueda S, Kanzaki T, Saito Y, Yoshida S. 1993. The phospholipase- $\mathrm{A}_{2}$ reaction leads to increased monocyte adhesion of endothelial cells via the expression of adhesion molecules. Eur J Biochem 217: 723-729.

16) Sakai M, Miyazaki A, Hakamata H, Sato Y, Matsumura T, Kobori S, Shichiri M, Horiuchi S. 1996. Lysophosphatidylcholine potentiates the mitogenic activity of modified LDL for human monocyte-derived macrophages. Arterioscl Thromb Vasc Biol 16: 600-605.

17) Sasaki Y, Asaoka Y, Nishizuka Y. 1993. Potentiation of diacylglycerol-induced activation of protein kinase $\mathrm{C}$ by lysophospholipids. Fed Eur Biochem Soc 320: 47-51.

18) Tokumura A, Harada K, Fukuzawa K, Tsukatani H. 1986. Involvement of lysophospholipase D in the production of lysophosphatidic acid in rat plasma. Biochim Biophys Acta 875: 31-38.

19) Eichholts T, Jalink K, Fahrenfort I, Moolenaar WH. 1993. The bioactive phospholipid lysophosphatidic acid is released from activated platelet. Biochem J 291: 677-680.

20) Cases A, Reverter JC, Escolar G, Sanz C, Sorribes J, Ordinas A. 1997. In vivo evaluation of platelet activation by different cellulosic membranes. Artif Organs 21: 330-334.

21) Nishizawa Y, Shoji T, Kawagishi T, Morii H. 1997. Atherosclerosis in uremia: possible roles of hyperparathyroidism and intermediate density lipoprotein accumulation. Kidney Int 52: S90-S92.

22) Goldstein DJ, Wheeler DC, Salant DJ. 1996. Effects of omega-3 fatty acids on complement-mediated glomerular epithelial cell injury. Kidney Int 50: 1863-1871.

23) Yalçin AS, Yurtkuran M, Dilek K, Kilinç A, Taga Y, Emerk K. 1989. The effect of vitamin $\mathrm{E}$ therapy on plasma and erythrocyte lipid peroxidation in chronic hemodialysis patients. Clin Chim Acta 185: 109-112.

24) Toborek M, Wasik T, Drózdz M, Magner-Wróbel K, Kopienczna-Grzebieniak E. 1992. Effect of hemodialysis on lipid peroxidation and antioxidant system in patients with chronic renal failure. Metabolism 41: 1229-1232.

25) Anderson JG, Thomas TH, Wilkinson R. 1996. Increased susceptibility to membrane lipid peroxidation in renal failure. Nephron 74: 373-377. 